\title{
Obstacle crossing among people with Parkinson disease is influenced by concurrent music
}

\author{
Lesley A. Brown, PhD; ${ }^{*}$ Natalie de Bruin, MSc; ${ }^{1}$ Jon Doan, PEng, PhD; ${ }^{1}$ Oksana Suchowersky, MD; ${ }^{2-3}$ \\ Bin Hu, PhD, MD $^{2}$ \\ ${ }^{1}$ Balance Research Laboratory, Department of Kinesiology, University of Lethbridge, Lethbridge, Canada; Departments \\ of ${ }^{2}$ Clinical Neurosciences and ${ }^{3}$ Medical Genetics, University of Calgary, Calgary, Canada
}

\begin{abstract}
Multitasking situations exacerbate gait impairments and increase the risk of falling among people with Parkinson disease (PD). This study compared obstacle negotiation among 10 subjects with PD and 10 nonparkinsonian control (CTRL) subjects in two test conditions differentiated by the presence of music played through a personal music player. Subjects walked the length of a $10 \mathrm{~m}$ walkway at a selfselected pace, crossing a $0.15 \mathrm{~m}$ obstacle placed at the midpoint of the walkway. The results indicated that subjects with PD crossed the obstacle slower than CTRL subjects and that concurrent music differentially altered obstacle crossing behaviors for the CTRL subjects and subjects with PD. Subjects with PD further decreased obstacle-crossing velocities and maintained spatial parameters in the music condition. In contrast, CTRL subjects maintained all spatiotemporal parameters of obstacle crossing with music. The alterations to crossing behaviors observed among the subjects with PD support our previous suggestion that listening to music while walking may be an attentionally demanding task.
\end{abstract}

Key words: bradykinesia, cues, dual task, fall risk, gait, multitasking, music, obstacle negotiation, Parkinson disease, walking.

\section{INTRODUCTION}

People with Parkinson disease (PD) are at the highest risk of falling among individuals with neurological impairments [1], with 70 percent of people with PD reported to fall annually and 50 percent experiencing multiple falls each year [1-2]. Balance impairments and cognitive deficits have been identified as the leading independent intrin- sic (patient-oriented) risk factors [3-4], while obstacle avoidance [5] and dual or multitasking situations [6] have been implicated as the foremost extrinsic (environmental) risk factors for falls in the population with PD. The decrement to gait performance observed when individuals with PD execute multiple tasks simultaneously, and the implied threat to stability, has led to the development of multitask training protocols [7-9]. These training protocols aim to increase functionality through improving the individual's capacity to perform additional tasks concurrent to walking.

Our recent work exploring the effects of concurrent music on steady-state parkinsonian gait has provided further support regarding the dual and multitask limitations experienced by people with PD [10]. Our use of contemporary commercial music closely simulated a real-world scenario in which walking with music has become a phenomenon that is encountered on a daily basis. Our findings

Abbreviations: $\mathrm{CTRL}=$ control, $\mathrm{CVCOM}=$ crossing velocity of whole-body center of mass, CVLead = crossing velocity of lead limb, CVTrail = crossing velocity of trail limb, ES = effect size, $\mathrm{HO}=$ heel-obstacle distance, $\mathrm{PD}=$ Parkinson disease, SHLead = step height of lead foot, SHTrail = step height of trail foot, $\mathrm{SL}=$ step length, $\mathrm{TO}=$ toe-obstacle distance.

* Address all correspondence to Lesley A. Brown, PhD; Balance Research Laboratory, Department of Kinesiology, University of Lethbridge, 4401 University Drive, Lethbridge, Alberta, Canada T1K 3M4; 403-382-7181; fax: 403380-1839. Email: 1.brown@uleth.ca

DOI:10.1682/JRRD.2009.10.0171 
indicated that while control (CTRL) subjects did not demonstrate a change in spatiotemporal parameters of gait, subjects with PD walked differently when listening to music [10]. Specifically, subjects with PD spent a significantly longer proportion of the gait cycle in double-limb support during walking trials in which they were listening to music. When a multitask scenario was imposed in which subjects were asked to perform an arithmetic problem concurrent to walking and listening to music, subjects with PD demonstrated slower gait velocity, a further increase in doublelimb support duration, and shorter strides, while the gait of CTRL subjects was unaltered. We suggested from these findings that listening to music while walking may be an attention-demanding activity for people with PD [10].

The motoric and attentional demands of locomotion in complex everyday environments typically exceed those of steady-state gait [11]. In dealing with these demands, it would be valuable for the management of parkinsonian gait deficits to develop multitask training protocols that extend beyond the relative simplicity of steady-state gait to include functional gait activities. Obstacle crossing, an example of a functional gait activity, has been identified as an external risk factor for falls among neurological patients, and successful obstacle crossing is associated with safe navigation of the daily environment [1]. Accordingly, the primary purpose of this study was to explore whether concurrent music affects the obstacle crossing behaviors of nonparkinsonian subjects and subjects with PD. Curiously, despite the identification of impaired obstacle negotiation as a major cause of falls among neurological patients [1], the behaviors associated with obstacle negotiation among people with PD are presently unclear. Consequently, the research question addressed in this article also provided an opportunity to contribute further knowledge and define the obstacle negotiation kinematics of people with PD while crossing a threedimensional obstacle. In line with our previous work, we hypothesized that an expected decrement in obstacle negotiation kinematic parameters among subjects with PD would be further exacerbated when these subjects concurrently listened to music [10].

\section{METHODS}

\section{Subjects}

We included a total of 20 subjects in this study10 subjects with idiopathic PD (subject characteristics in Table 1) and 10 age-matched, nonparkinsonian CTRL sub-
Table 1.

Subject characteristics.

\begin{tabular}{lccc}
\hline \multicolumn{1}{c}{ Subjects } & CTRL & PD & p-Value \\
\hline Demographics & 10 & 10 & - \\
No. of Subjects & $65.9 \pm 6.0$ & $67.2 \pm 6.1$ & 0.63 \\
Age (yr, mean \pm SD) & $3 / 7$ & $7 / 3$ & 0.07 \\
Sex (M/F) & $1.67 \pm 0.10$ & $1.69 \pm 0.10$ & 0.69 \\
Height (m, mean \pm SD) & & & \\
Clinical Characteristics & & $4.2 \pm 7.3$ & - \\
$\quad$ (mean \pm SD) & NA & $2.5 \pm 0.3$ & - \\
Disease Duration (yr) & NA & $27.3 \pm 5.3$ & - \\
Hoehn and Yahr & NA & \\
UPDRS (III) & Note: Values are mean \pm standard deviation (SD) for continuous variables and \\
number for nominal variables. Hoehn and Yahr and UPDRS (III) scores were \\
measured with patients on antiparkinsonian medications. \\
CTRL = control, F = female, M = male, NA = not applicable, PD = Parkinson \\
disease, UPDRS = Unified Parkinson Disease Rating Scale (III). \\
\hline \hline
\end{tabular}

jects. We recruited all subjects from the local community, recruiting the subjects with PD through local support groups and neurologists. We informed subjects of the nature of the study and received their informed consent before testing. We included subjects in the study if they had been diagnosed with idiopathic PD by a consultant neurologist, had mild to moderate disease severity as determined by the Hoehn and Yahr scale (Stage II to III) [12], had a stable medication regimen (a minimum of 1 month before testing), and had the ability to ambulate independently without using a walking aid. We excluded subjects with PD if they had a disease duration of $<1$ year, had an additional neurological disorder or comorbidity likely to affect gait (e.g., stroke, orthopedic disease, diabetes mellitus), had a score of $\leq 26$ on the Mini-Mental Status Examination [13], had a hearing deficit, or were already walking to music. We included CTRL subjects if they did not have a hearing deficit, could ambulate independently without the use of a walking aid, and were free of any self-reported neurological disorders or comorbidities that would affect gait (e.g., stroke, orthopedic disease, diabetes mellitus).

\section{Protocol}

Subjects walked the length of a $10 \mathrm{~m}$ walkway at a self-determined pace in six different testing conditions. We distinguished test conditions by music accompaniment (no music/music), asking the subjects to perform a concurrent arithmetic task (single task/dual task), and obstacle (no obstacle/obstacle). The dataset in this article addresses obstacle negotiation trials. We addressed singleand dual-task trials in a separate report because of the 
differences in motor patterning between steady-state gait and obstacle negotiation [10].

Subjects performed 6 obstacle negotiation trials in each music condition for a total of 12 trials. We randomized task order to control for order and/or practice effects. We blocked trials by the presence of music and counterbalanced the presentation order of the blocks between subjects. Subjects performed a practice trial without music before data collection. We completed all testing with the subjects with PD on medication, testing a minimum of 1 hour postmedication. A trained researcher walking behind each subject ensured safety.

\section{Apparatus}

We placed reflective markers on the sternal notch and bilaterally on the acromion process, lateral humeral epicondyle, ulnar styloid process, greater trochanter, lateral femoral condyle, lateral malleolus, the anterior aspect of the shoe (approximately between the first and second metatarsal), and the posterior aspect of the shoe (in the region of the calcaneus). We collected three-dimensional kinematic data using a six-camera motion analysis system $(120 \mathrm{~Hz}$; Pulnix TM-6701AN progressive scan camera system [Pulnix America, Inc; Sunnyvale, California] and Vicon Motus 9.2 software [VICON; Englewood, Colorado]).

The obstacle was a dense foam block $(0.15 \mathrm{~m}$ high $\times$ $0.15 \mathrm{~m}$ deep $\times 0.60 \mathrm{~m}$ wide) placed at the midpoint of the walkway before trial onset. We marked the obstacle with a retroreflective marker placed at the top/center of the sagittal face. We played music at a self-selected volume through headphones with an iPod Nano ${ }^{\circledR}$ (Apple, Inc; Cupertino, California), which we attached to participants' waistbands. We captured frontal and sagittal video using digital video cameras.

\section{Measures of Interest}

In accordance with convention, we designated the first leg to cross the obstacle as the lead limb and the second leg to cross the obstacle as the trail limb. Subjects were free to self-select the crossing limb for all trials. The spatiotemporal parameters of obstacle crossing we calculated for each trial were step length (SL); toe-obstacle distance (TO); heel-obstacle distance (HO); step height of lead foot (SHLead) and trail foot (SHTrail); and crossing velocity of the lead limb (CVLead), trail limb (CVTrail), and whole-body center of mass (CVCOM). We defined SL as the mean horizontal distance between the toe-off position and heel-contact position for the lead and trail feet during obstacle crossing. We described the TO as the horizontal distance between the toe-off position of the trail foot and the front edge of the obstacle before obstacle crossing and the $\mathrm{HO}$ as the horizontal distance between the rear edge of the obstacle and the heel-contact position of the lead foot following obstacle crossing. We defined SHLead and SHTrail as the vertical distance between the toe of the foot and the center of the top surface of the obstacle during the obstacle crossing step. Figure 1 illustrates the spatial measures of obstacle negotiation included in this study.

\section{Data Processing}

We interpolated raw marker data as necessary and filtered it at $10 \mathrm{~Hz}$ using a low-pass fourth-order Butterworth filter. We processed filtered marker data and calculated spatiotemporal parameters of gait using custom written algorithms (MATLAB ${ }^{\circledR}$ version R2007a, The MathWorks; Natick, Massachusetts). We compared data between trials to determine whether performance in successive trials was influenced by practice or fatigue. We did not identify an effect for order; therefore, we calculated mean values for each dependent variable from six obstacle crossing trials in each testing condition (no music/music).

We selected music based on the genre and artist preferences of the subject, determined through a prior telephone interview. We determined the properties of each subject's music selections independently by two raters. We used the presence of vocal content in each musical track to differentiate the track as lyrical or instrumental. We established the tempo of each track using Jackson 1.34 DJ sequencing software (Van Aeken Software; Brussels, Belgium).

We determined freezing events using the sagittal video records. We identified a freezing event as an interruption in forward progression during locomotion. A custom written algorithm set to identify a change in the static position of the obstacle detected obstacle contact occurrences. We confirmed obstacle contact events to occur as a result of contact between foot and obstacle from the sagittal video record.

\section{Statistical Analysis}

We analyzed data using SPSS 17.0 for Windows (SPSS Inc; Chicago, Illinois). We summarized demographic data and music properties descriptively and compared them between groups using independent $t$-tests and chi-square tests. We analyzed the effect of Music and Group on spatiotemporal parameters of obstacle negotiation using separate mixed 2-factor (Group [CTRL/PD] × Music [no music/music]) repeated-measures analyses of 


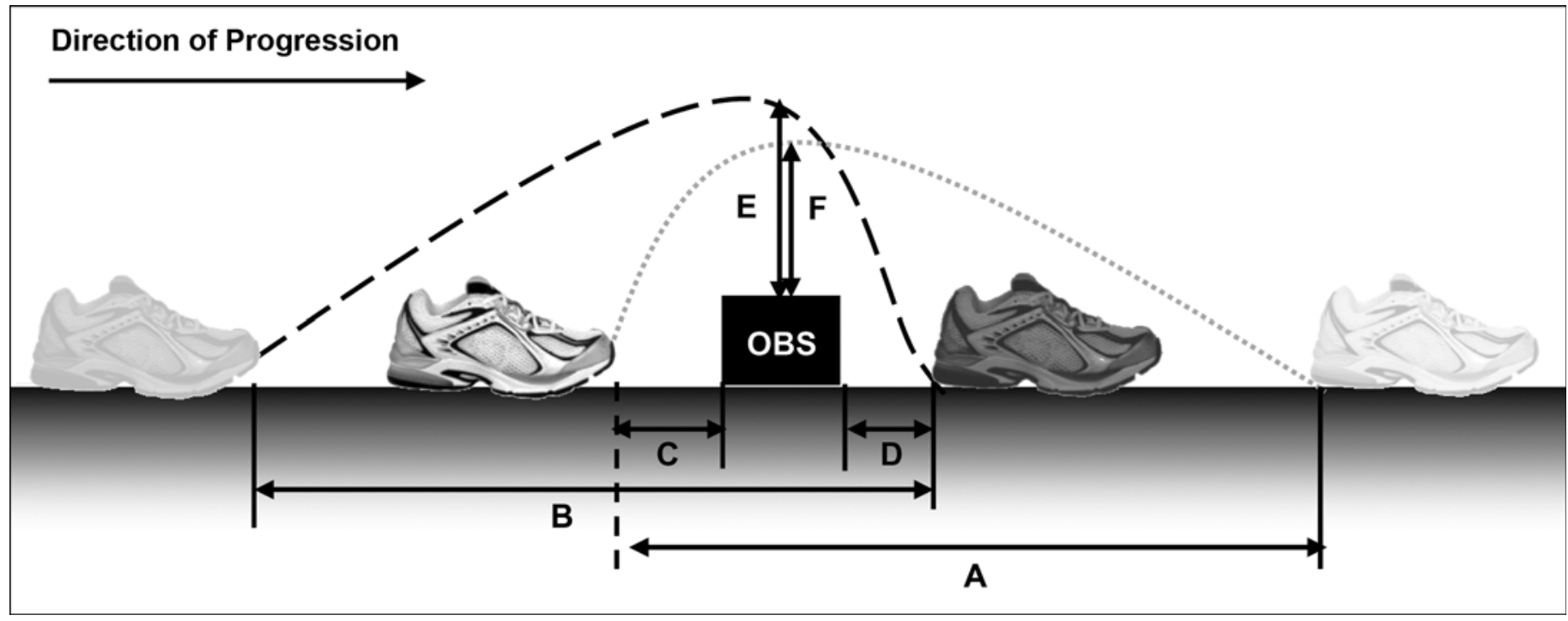

Figure 1.

Spatial measures of interest. Gray fill indicates lead limb foot; light fill indicates trail limb foot. Dark dashed line indicates approximate trajectory of lead limb foot; light dotted line indicates approximate trajectory of trail limb foot. Measures shown are (A) step length of lead foot, (B) step length of trail foot, (C) toe-obstacle distance, (D) heel-obstacle distance, (E) step height of lead foot, and (F) step height of trail foot. Temporal measures, crossing velocity of lead limb, crossing velocity of trail limb, and crossing velocity of center of mass are not shown. OBS $=$ obstacle.

variance. We set statistical significance at 0.05 . We reported effect size (ES) with partial $\eta^{2}$ values.

\section{RESULTS}

Subject characteristics were similar for CTRL subjects and subjects with PD (Table 1). The mean tempo of the music selections was consistent between groups $(t(18)=0.101, p=0.92$; CTRL $=105.6$ beats per minute; $\mathrm{PD}=105.0$ beats per minute) as confirmed by two independent raters (intraclass correlation coefficient = 0.860 ; $95 \%$ confidence intervals $0.751-0.924$ ). In addition, the lyrical content of the tracks was comparable between groups $(t(18)=0.152, p=0.88$; CTRL $=80.0 \%$; $\mathrm{PD}=77.1 \%)$. The incidence of obstacle contacts did not differ between groups or conditions, with zero obstacle contacts recorded. In addition, we observed zero freezing episodes for subjects with PD.

Table 2 provides descriptive statistics and summary statistical findings for spatiotemporal obstacle negotiation parameters. When concurrently listening to music, the CTRL subjects and subjects with PD differentially altered a number of temporal obstacle crossing parameters. Specifically, in the music trials, subjects with PD crossed the obstacle slower than in the no music condition, a finding that was demonstrated by a 7.2 percent decrease in CVLead and a 7.4 percent decrease in CVCOM in the music condition. In contrast, CTRL subjects largely maintained obstacle crossing velocity during music trials (CVLead, 0.1\% increase; CVCOM, 0.5\% decrease). We confirmed this differential effect by a significant interaction between Music and Group for CVLead $(F(1,18)=5.242, p=0.03$; ES = 0.226; Figure 2(a)), and an interaction approaching significance for CVCOM $(F(1,18)=4.212, p=0.06$; $\mathrm{ES}=0.190$; Figure 2(b)). SL, TO, HO, SHLead, SHTrail, and CVTrail were maintained between no music and concurrent music trials for both CTRL subjects and subjects with PD ( $p>$ 0.05). Furthermore, the obstacle crossing behavior of the subjects with PD was consistently defined by a significantly slower CVLead $(F(1,18)=6.650, p=0.02$; ES = 0.270 ; Figure 2(a)) and CVCOM $(F(1,18)=5.792, p=$ 0.03; ES $=0.243$; Figure 2(b)) when compared with the CTRL group.

\section{DISCUSSION}

The primary purpose of the current study was to establish the effect of concurrent music on the obstacle 
Table 2.

Summary of descriptive statistics and statistical findings for gait parameters in group with Parkinson disease (PD) and control (CTRL) group.

\begin{tabular}{|c|c|c|c|c|c|c|c|c|c|}
\hline \multirow{2}{*}{ Parameter } & \multicolumn{3}{|c|}{ CTRL } & \multicolumn{3}{|c|}{ PD } & \multirow{2}{*}{ G } & \multirow{2}{*}{$\mathbf{M}$} & \multirow{2}{*}{$\mathbf{M} \times \mathbf{G}$} \\
\hline & NM & $\mathbf{M}$ & Change & NM & $\mathbf{M}$ & Change & & & \\
\hline Step Length (m) & $0.866 \pm 0.18$ & $0.862 \pm 0.14$ & 0.004 (-0.04 to 0.05$)$ & $0.851 \pm 0.14$ & $0.831 \pm 0.14$ & 0.020 (-0.06 to 0.10$)$ & 0.730 & 0.549 & 0.701 \\
\hline Heel-Obstacle Distance (m) & $0.176 \pm 0.05$ & $0.181 \pm 0.05$ & 0.005 (-0.03 to 0.02$)$ & $0.155 \pm 0.05$ & $0.156 \pm 0.06$ & $-0.001(-0.02$ to 0.02$)$ & 0.275 & 0.746 & 0.814 \\
\hline Step Height of Lead Foot (m) & $0.182 \pm 0.04$ & $0.179 \pm 0.04$ & 0.004 ( -0.01 to 0.02$)$ & $0.186 \pm 0.01$ & $0.193 \pm 0.03$ & -0.007 ( -0.03 to 0.01$)$ & 0.526 & 0.714 & 0.241 \\
\hline Step Height of Trail Foot (m) & $0.155 \pm 0.05$ & $0.155 \pm 0.04$ & 0.0002 (-0.02 to 0.02$)$ & $0.136 \pm 0.03$ & $0.146 \pm 0.04$ & $-0.010(-0.02$ to 0.01$)$ & 0.422 & 0.355 & 0.377 \\
\hline Velocity of COM (m/s) & $1.125 \pm 0.10$ & $1.119 \pm 0.10$ & $0.006(-0.04$ to 0.05$)$ & $1.032 \pm 0.14$ & $0.956 \pm 0.15$ & $0.076(0.01$ to 0.04$)$ & 0.027 & 0.027 & 0.055 \\
\hline
\end{tabular}

(a)

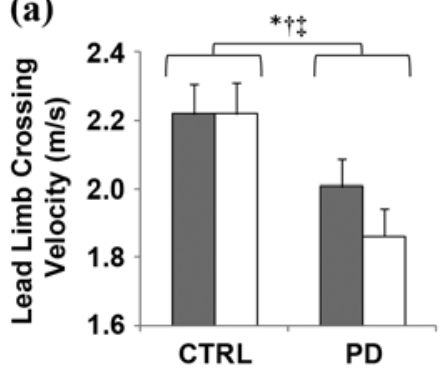

(b)

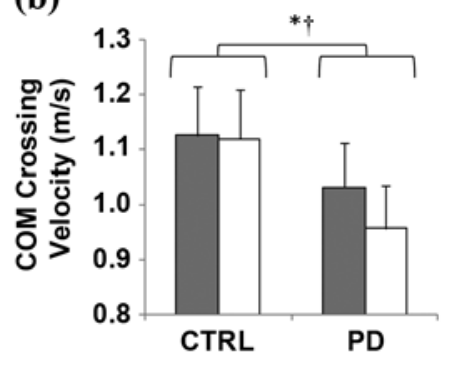

Figure 2.

Results of kinematic analysis of (a) lead limb and (b) center of mass (COM) crossing velocity in control (CTRL) subjects and subjects with Parkinson disease (PD). Dark bars represent no music trials, while light bars represent music trials. Data presented are mean and standard error of mean. * Significant effect of music. ${ }^{\dagger}$ Significant effect of group. ${ }^{\ddagger}$ Significant music $\times$ group interaction.

crossing behaviors of subjects with PD and nonparkinsonian CTRL subjects. To accomplish this goal, it was first necessary to ascertain the movement patterns of people with PD when crossing a three-dimensional obstacle. Subjects with PD and CTRL subjects were able to safely avoid obstacle contact during crossing. In this study, the obstacle was visible from the outset of the trial, evidently allowing subjects adequate time to modify the spatial and temporal characteristics of their stepping behavior to successfully and safely negotiate the obstruction. Subjects with PD crossed the obstacle considerably slower (CVLead and CVCOM) than CTRL subjects, even in the no music condition. To address the possibility that parkinsonian bradykinesia was exacerbated by the presence of an obstacle, we conducted a post hoc analysis of
CVCOM using data obtained from no music trials of unobstructed (no obstacle) and obstructed (obstacle) walking trials. Analysis revealed equivalent decreases in velocity among CTRL subjects and subjects with PD $(\mathrm{CTRL}=17.9 \%, \mathrm{PD}=18.2 \% ; t(18)=0.113, p=0.91)$ when we obstructed walking with an obstacle. Based on this finding, we suggest that the observed impairment in obstacle crossing velocity displayed by the subjects with PD reflects the bradykinesia that is inherent amongst the population with PD during steady-state locomotion [14-15] and does not reflect exacerbated bradykinesia in the presence of an obstruction.

Adding music to the task of obstacle negotiation differentially influenced the obstacle-crossing behaviors of the CTRL subjects and subjects with PD. More specifically, CTRL subjects maintained spatiotemporal parameters of obstacle negotiation with the addition of music. Conversely, and in concord with our hypothesis, subjects with PD demonstrated a further decrease in obstacle crossing velocities (CVLead and CVCOM) when concurrently listening to music; spatial parameters of obstacle crossing were, however, preserved. Slower obstacle crossing velocities in nonparkinsonian older adults have been associated with a conservative crossing strategy, allowing subjects greater opportunity for recovery in the event of obstacle contact [16-17]. In the population with PD, however, it may be more appropriate to interpret reduced obstaclecrossing velocities as representing a detrimental obstaclecrossing behavior, as the decreased crossing speed may result in more time being spent in the unstable singlesupport phase of the gait cycle. This contrasts the behavior of people with PD during unobstructed walking, where 
patients significantly increase the amount of time spent in double-limb support [18] to maintain stability. Similar to our previous work, we suggest that the decrease in obstaclecrossing velocity observed in this study may reflect dualtask interference [10]. We postulate that listening to selfselected music concurrent to walking acts as an additional "task," diverting attentional resources away from the primary task of walking and safely negotiating an obstruction. Despite this inference, in the experimental context provided in this study, subjects with PD were still capable of making sufficient online adjustments to gait patterning to ensure safe and successful negotiation of an obstacle in a situation that presented auditory distraction.

The majority of falls in the parkinsonian population are reported when patients are on medications and feel that their symptoms are well managed [19]. Consequently, it was first necessary to describe the obstacle-crossing behaviors of people with PD in the medicated condition. Therefore, the results presented in this article should not be generalized to situations where patients are "off" medications or experiencing fluctuations in medication status. In addition, the small, relatively homogeneous sample population recruited for this study limits the generalizability of our findings to the wider patient community in which symptoms and disease severity are more heterogeneous. A sex disparity existed between the subjects with PD and CTRL subjects $(p=0.07)$. Since SL has been reported to be shorter among female older adults [20], the sex inequality between groups could conceivably contribute to the lack of differences observed in spatial parameters of obstacle crossing. Furthermore, females tend to walk with a slower walking speed [21], potentially reducing the differences in obstacle-crossing velocities reported between groups. We propose that future research should incorporate increasingly challenging obstacle-crossing tasks, such as the presence of a time constraint or a secondary task to more closely mimic natural walking environments. In addition, joint patterning and kinetic parameters of obstacle crossing could be examined to offer a more thorough description of the obstacle negotiation behaviors of people with PD. This insight may help resolve the elevated fall risk experienced by this population.

\section{CONCLUSIONS}

While commonalities exist between the obstaclecrossing behaviors displayed by subjects with PD and CTRL subjects, the crossing behavior demonstrated by the subjects with PD reflects bradykinetic gait patterning that was not exacerbated by the presence of an obstacle. The addition of concurrent music to the obstacle-negotiation task did, however, further decrease obstacle crossing velocity among subjects with PD. Based on current evidence, we have interpreted the alterations to crossing behaviors exhibited by subjects with PD while concurrently listening to music to represent dual-task interference. These findings have implications for patient safety during complex obstacle-crossing scenarios that are commonly encountered during activities of daily living. To this end, future gait training interventions aimed at improving functional ambulation and reducing fall risk among people with PD should integrate the performance of obstacle negotiation tasks accompanied by a variety of environmental distracters.

\section{ACKNOWLEDGMENTS}

\section{Author Contributions:}

Conception and design of study: L. A. Brown, J. Doan, O. Suchowersky, B. Hu.

Provision of study materials and analysis tools: L. A. Brown, J. Doan. Collection of data: L. A. Brown, N. de Bruin, J. Doan.

Analysis and interpretation of data: L. A. Brown, N. de Bruin, J. Doan. Drafting of manuscript: L. A. Brown, N. de Bruin.

Critical revision of manuscript for important intellectual content: J. Doan, O. Suchowersky, B. Hu.

Final approval of manuscript: L. A. Brown, B. Hu.

Financial Disclosures: The authors have declared that no competing interests exist.

Funding/Support: This material was based on work supported by the Canadian Institutes of Health Research (grant 79025).

Institutional Review: The Human Research Ethics Committee of the University of Lethbridge provided ethical approval of the study.

Participant Follow-Up: The authors plan to inform participants of the publication of this article.

\section{REFERENCES}

1. Stolze H, Klebe S, Zechlin C, Baecker C, Friege L, Deuschl G. Falls in frequent neurological diseases-Prevalence, risk factors and aetiology. J Neurol. 2004;251(1):79-84. [PMID: 14999493] DOI:10.1007/s00415-004-0276-8

2. Bloem BR, Hausdorff JM, Visser JE, Giladi N. Falls and freezing of gait in Parkinson's disease: A review of two interconnected, episodic phenomena. Mov Disord. 2004;19(8): 871-84. [PMID: 15300651] DOI:10.1002/mds.20115 
3. Wood BH, Bilclough JA, Bowron A, Walker RW. Incidence and prediction of falls in Parkinson's disease: A prospective multidisciplinary study. J Neurol Neurosurg Psychiatry. 2002; 72(6):721-25. [PMID: 12023412]

DOI:10.1136/jnnp.72.6.721

4. Ashburn A, Stack E, Pickering RM, Ward CD. A communitydwelling sample of people with Parkinson's disease: Characteristics of fallers and non-fallers. Age Ageing. 2001;30(1): 47-52. [PMID: 11322672] DOI:10.1093/ageing/30.1.47

5. Robinson K, Dennison A, Roalf D, Noorigian J, Cianci H, Bunting-Perry L, Moberg P, Kleiner-Fisman G, Martine R, Duda J, Jaggi J, Stern M. Falling risk factors in Parkinson's disease. NeuroRehabilitation. 2005;20(3):169-82. [PMID: 16340098]

6. Willemsen MD, Grimbergen YA, Slabbekoorn M, Bloem BR. [Falling in Parkinson disease: More often due to postural instability than to environmental factors]. Ned Tijdschr Geneeskd. 2000;144(48):2309-14. Dutch. [PMID: 11143299]

7. Bedeschi C, Ibrahim J, Cardoso CA, Loureiro BD, Okamoto E, Ribeiro do Valle LE, Piemonte ME. Effects of a new approach to improve the gait performance in dual-task conditions for patients with Parkinson's disease. Parkinsonism Relat Disord. 2008;14:S58. DOI:10.1016/S1353-8020(08)70288-8

8. Canning CG, Ada L, Woodhouse E. Multiple-task walking training in people with mild to moderate Parkinson's disease: A pilot study. Clin Rehabil. 2008;22(3):226-33.

[PMID: 18285432] DOI:10.1177/0269215507082341

9. Piemonte ME, Okamoto L, Richi L, Valle LE. Is it possible to train the ability to divide the attention during gait of patients with Parkinson's disease? Parkinsonism Relat Disord. 2008; 14:S65-66. DOI:10.1016/S1353-8020(08)70318-3

10. Brown LA, De Bruin N, Doan JB, Suchowersky O, Hu B. Novel challenges to gait in Parkinson's disease: The effect of concurrent music in single- and dual-task contexts. Arch Phys Med Rehabil. 2009;90(9):1578-83. [PMID: 19735787] DOI:10.1016/j.apmr.2009.03.009

11. Siu KC, Catena RD, Chou LS, Van Donkelaar P, Woollacott $\mathrm{MH}$. Effects of a secondary task on obstacle avoidance in healthy young adults. Exp Brain Res. 2008;184(1):115-20. [PMID: 17717655]

DOI:10.1007/s00221-007-1087-9
12. Hoehn MM, Yahr MD. Parkinsonism: Onset, progression, and mortality. 1967. Neurology. 2001;57(10 Suppl 3):S11-26. [PMID: 11775596]

13. Folstein MF, Folstein SE, McHugh PR. "Mini-mental state.” A practical method for grading the cognitive state of patients for the clinician. J Psychiatr Res. 1975;12(3):189-98.

[PMID: 1202204] DOI:10.1016/0022-3956(75)90026-6

14. Blin O, Ferrandez AM, Serratrice G. Quantitative analysis of gait in Parkinson patients: Increased variability of stride length. J Neurol Sci. 1990;98(1):91-97. [PMID: 2230833] DOI:10.1016/0022-510X(90)90184-O

15. Morris ME, Iansek R, Matyas TA, Summers JJ. The pathogenesis of gait hypokinesia in Parkinson's disease. Brain. 1994;117(Pt 5):1169-81. [PMID: 7953597] DOI:10.1093/brain/117.5.1169

16. Galna B, Peters A, Murphy AT, Morris ME. Obstacle crossing deficits in older adults: A systematic review. Gait Posture. 2009;30(3):270-75. [PMID: 19625191] DOI:10.1016/j.gaitpost.2009.05.022

17. Patla AE, Prentice SD, Robinson C, Neufeld J. Visual control of locomotion: Strategies for changing direction and for going over obstacles. J Exp Psychol Hum Percept Perform. 1991;17(3):603-34. [PMID: 1834781] DOI:10.1037/0096-1523.17.3.603

18. Knutsson E. An analysis of Parkinsonian gait. Brain. 1972; 95(3):475-86. [PMID: 4655275] DOI:10.1093/brain/95.3.475

19. Bloem BR, Grimbergen YA, Cramer M, Willemsen M, Zwinderman AH. Prospective assessment of falls in Parkinson's disease. J Neurol. 2001;248(11):950-58. [PMID: 11757958] DOI:10.1007/s004150170047

20. Laufer Y. Age- and gender-related changes in the temporalspatial characteristics of forwards and backwards gaits. Physiother Res Int. 2003;8(3):131-42. [PMID: 14533369] DOI:10.1002/pri.281

21. Richard R, Weber J, Mejjad O, Polin D, Dujardin F, Pasquis P, Le Loët X. Spatiotemporal gait parameters measured using the Bessou gait analyzer in 79 healthy subjects. Influence of age, stature, and gender. Study Group on Disabilities due to Musculoskeletal Disorders (Groupe de Recherche sur le Handicap de l'Appareil Locomoteur, GRHAL). Rev Rhum Engl Ed. 1995;62(2):105-14. [PMID: 7600064]

Submitted for publication October 21, 2009. Accepted in revised form January 14, 2010. 
